\title{
Design and Implementation of Fire Safety Education System on Campus based on Virtual Reality Technology
}

\author{
Kun Zhang, Jintao Suo, Jingying Chen, Xiaodi Liu, Lei Gao \\ National Engineering Research Center for E-Learning, Central China Normal University, China \\ Email: zhk@mail.ccnu.edu.cn, chenjy@mail.ccnu.edu.cn
}

\begin{abstract}
Fire safety education is essential to every student on campus. Fire safety knowledge learning and operational practice are both important. There is evidence that the virtual reality (VR) based educational method can be a novel and effective approach to learning and practice. However, the existing VR-based system for fire safety education has some shortcomings such as lack of interactivity and high equipment complexity, resulting in low practicability. In order to improve the effect of fire safety education on campus, this paper establishes the model and architecture of fire safety education system based on VR technology. The framework and various elements of fire safety education system are designed and implemented according to the combination of relevant fire safety education theory and VR technology. Finally the prototype version of fire safety education system based on VR technology is built on the HTC VIVE helmet equipment. Through the usability test and comparative analysis of the application experiment, the experiment results prove the feasibility and effectiveness of the proposed approach.
\end{abstract}

\section{INTRODUCTION}

$\mathrm{T}$ HE fire is a major disaster that threatens human safety. Due to lack of safety knowledge, improper emergency measures and so on, the campus is prone to fire. The colleges and universities must attach great importance to fire safety education. Each student must learn and master the fire safety knowledge and necessary skills to prevent the fire and handle properly in the event of fire [1]. This is of great significance for maintaining the campus order and the safety of students.

Fire safety education emphasizes the close combination of knowledge learning and operational practice. It is important to choose the appropriate teaching method [2], which will directly affect the teaching effect.

The traditional teaching methods are mainly textbook teaching or multimedia presentation. Both of these methods have the shortcomings of poor interaction and lack of practical exercise. Fire drills can effectively help students become familiar with firefighting equipment and learn extinguishing and self-protection skills [3]. However, due to the constraints of material, manpower and money, fire drills cannot be carried out frequently, which may lead to the decline in students' fire safety ability.

This work was supported by the Research Funds of CCNU from the Colleges' Basic Research and Operation of MOE (No. CCNU17QN0002).
With the emergence of virtual reality (VR) technology, it is widely used in game development, virtual training [4], and introduced into the field of education [5]. VR technology can provide a realistic virtual environment, allowing students to have the flow feeling with multisensory experience [6]. VR technology has also been introduced into the fire safety education. Bhagat [7] designs a cost-effective 3D VR system for military live fire training. $\mathrm{Xu}$ [8] establishes a VR-based fire training simulator with smoke assessment capability. On the whole, current research and applications are mainly focused on firefighting programs and evacuation plans. Especially, there are still some shortcomings in the VR-based fire safety education system. They are not in-depth integration with educational theory. The lack of interactivity and high equipment complexity also lead to the low practicability [9]. They cannot make full use in the practical fire safety education and teaching process.

Therefore, in order to improve the effect of fire safety education on campus, this paper establishes the design model and architecture of fire safety education system based on VR technology. The framework and various elements of fire safety education system are designed and implemented according to the combination of relevant educational theory and VR technology. Finally, VR-based fire safety education system is built on HTC VIVE helmet equipment. The system is applied to fire safety education, to help students learn fire safety knowledge and improve fire safety skills.

\section{SYSTEM DESIGN}

The design of fire safety education system fully integrates the relevant educational theory with VR technology, and follows the five design concepts. Thus, the system model and architecture are established.

\section{A. Relevant Educational Theory}

\section{1) Constructivism Learning Theory}

Constructivism theory advocates situational teaching. The student's knowledge is obtained through the construction of meaning in certain learning environments, with the aid of the necessary information and interaction. VR technology can create a virtual fire safety education environment similar to the actual situation. It provides students with the situational 
experience to learn the fire safety teaching content and to carry out fire safety interactive operation, which is conducive to students' construction of knowledge.

2) Flow Theory

The flow theory describes the phenomenon that students are fully immersed in the current environment and enter the flow state, concentrating their attention on learning content and achieving good learning outcomes. VR technology can provide students with a more realistic situational experience. Students will be easy to enter the flow state. They are fully concerned about the fire safety learning content and get more profound operation experience that will deepen the cognition and understanding of fire safety.

3) Gamification Learning

The gamification emphasizes the combination of learning content and gamified elements. The core of gamification is interest and reward mechanism. Through the scientific and attractive reward mechanism, students will have a continuous learning motivation. VR-based fire safety education system is presented in the form of gamification. With the interesting reward mechanism, students improve the participation and learning interest, and get more efficient learning outcomes.

4) Transfer of Learning

Transfer of learning provides a theoretical basis for the acquired skills transfer between virtual environment and real environment. The firefighting devices in the VR environment are mapped to the equipment in real world. Through virtual interaction with firefighting devices, students can learn fire safety skills and obtain the operational experience that can be transferred to the real activities. Through experiments, the effectiveness of learning transfer can be verified.

\section{B. Design Concept}

1) Instructional Design

Fire safety teaching content is divided into two categories, fire safety knowledge and operational practice. Fire safety knowledge contains fire alarm, fire protection, fire hazards and so on. The operational practice includes firefighting, fire escape, etc. According to constructivism theory, the teaching content is presented in a variety of different virtual situations. Students can explore and interact in the virtual environment, so as to construct their own knowledge system.

\section{2) Authentic Design}

The authenticity of the virtual situations can help students construct knowledge, enter the flow state, and get a profound learning experience. In order to ensure the authenticity of the VR scene, the object modeling design is the key. The $3 \mathrm{ds}$ Max is used to design and implement a set of models such as fire extinguishers, hydrants and so on. Then these models will be imported into Unity platform with further editing and rendering, to make the VR scene more realistic and vivid.

3) Interactive Design

The system interface is designed based on the principle of unconscious, making the interaction in line with the natural behavior of students. The operation of the system is designed based on the concept of natural human-computer interaction to facilitate the natural interaction between students and VR scenes. Students enter the flow state, explore and interact with a variety of fire safety situations. Interactive feedback is provided to help correcting errors and constructing cognition. The necessary instructions will be pre-set so that students can understand the basic situation.

4) Interesting Design

Related researches show that the gamification can not only increase student's interest, but also increase the learning effect. Visual effects and music melodies can create a variety of atmosphere, resulting in emotional resonance to stimulate student interest. Challenging reward strategies are also very helpful. The formulation of reward strategy needs to consider three aspects: principle, timing and form. Only the proper rewards can inspire students' interest. Through the usability test, it can get feedback for further improvement.

\section{System Architecture}

The architecture of VR-based fire safety education system includes user module, scene teaching module, test module, database module and I/O Module.

1) The user module is used to register and modify the user's basic information.

2) The scene teaching module is the core of the system. A series of virtual campus scenes are constructed to show fire safety knowledge and operational practice. Students can roam in the virtual campus, free access to different virtual scenes for interactive experience, so as to learn knowledge and master fire safety skills.

3) The test module is mainly used for students to carry out fire safety knowledge assessment, in order to check the effect of learning. The test content is consistent with the fire safety knowledge in the scene teaching module.

4) The database module is used to store students' usage records and data information, mainly including test scores, time cost and so on. SQLite is used as the database engine.

5) The I/O Module describes the way of interacting with the system, including the interactive mode based on helmet display and wireless controller (HTC VIVE), as well as the traditional I/O modes (keyboard, mouse and monitor).

\section{SYSTEM IMPLEMENTATION}

\section{A. Scene Implementation}

Using 3ds Max software, and Unity development platform, the virtual fire safety education environment similar to the actual situation is implemented to demonstrate the teaching contents, including fire safety knowledge and operational practice. A series of virtual campus scenes are constructed, including classrooms, dormitories and so on. Students can roam in the virtual campus, voluntarily enter different virtual scenes to explore and interact, so as to learn and construct their own knowledge system.

1) Knowledge Learning 
Fire safety knowledge includes fire type, fire hazard, fire alarm, fire prevention and so on. The teaching content is set in various places on the virtual campus scene. A series of fire safety signs and equipment will appear on the roaming route. When students point to them, the corresponding knowledge will be presented with voice, video and other forms. When students roam into several dedicated classrooms, they will carry out the collective learning of fire safety knowledge, in the form of multimedia presentation and knowledge quiz. Students handle the controller to answer, as shown in Fig. 1.

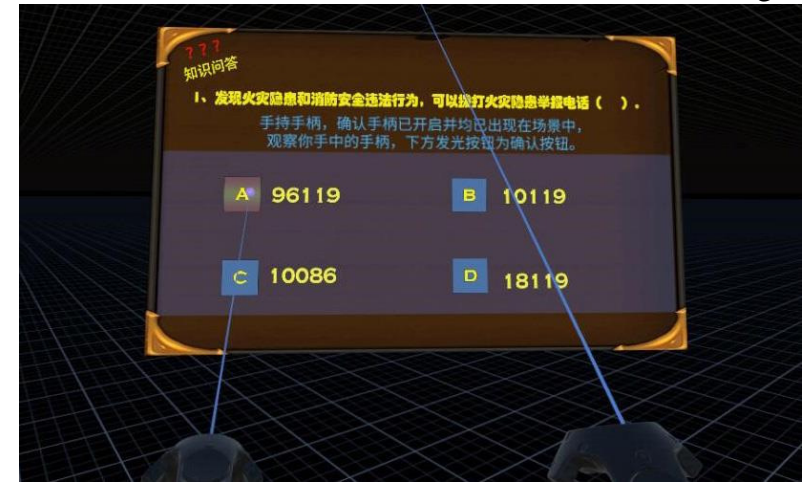

Fig. 1 The view of fire safety knowledge learning

\section{2) Operational Practice}

The realistic and vivid fire safety scenes are implemented and presented in front of students, enabling them to enter the flow state, get a profound experience and learn the fire safety operation skills that can be transferred to the real activities.

When students roam into certain specific scenes, they will trigger the corresponding fire drill tasks, including searching for fire points, fire extinguishing simulation, safe evacuation, etc. Students need to do the proper operation in virtual scenes, complete the fire practice task and get the reward.

One of the cases is to put out an office fire. The paper in the trash bin is ignited by a cigarette butt. The flame appears and continues to grow, accompanied by the burning sound. Following the prompts, the student need to pick up the fire extinguisher on the ground, operate it in accordance with the correct steps, and extinguish the fire, as shown in Fig. 2. After the task is completed, the office staff will come in, express gratitude and give bonus points. The accumulated points can be used to open more scenes.

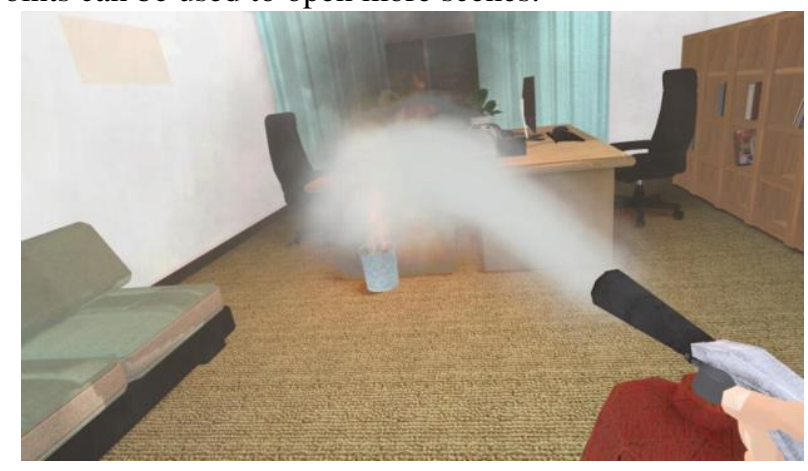

Fig. 2 The view of using fire extinguisher to put out a fire
Using VR scenes for virtual fire drills can overcome some limitations of traditional fire drills. More students can get the profound experience, and grasp the use of fire equipment. Subsequent experiments will verify the effect of transferring the skills acquired by virtual fire drills to the real activities.

\section{B. Interaction Implementation}

The interaction between students and the system is mainly based on HTC VIVE hardware platform. The kit consists of a helmet, two wireless controllers and two base stations for positioning. The VIVE controller is equipped with dual-stage trigger, 24 sensors, and has realistic haptic feedback. The base stations use Room-Scale positioning technology to track the exact locations of the helmet and controller with low latency and high accuracy. Wireless controllers in each hand combined with precise positioning and tracking mean that students can freely explore and interact with virtual objects, characters and environments, with the most natural behavior such as move forward, back off, squat, head rotation, grab and release and so on, as shown in Fig. 3.

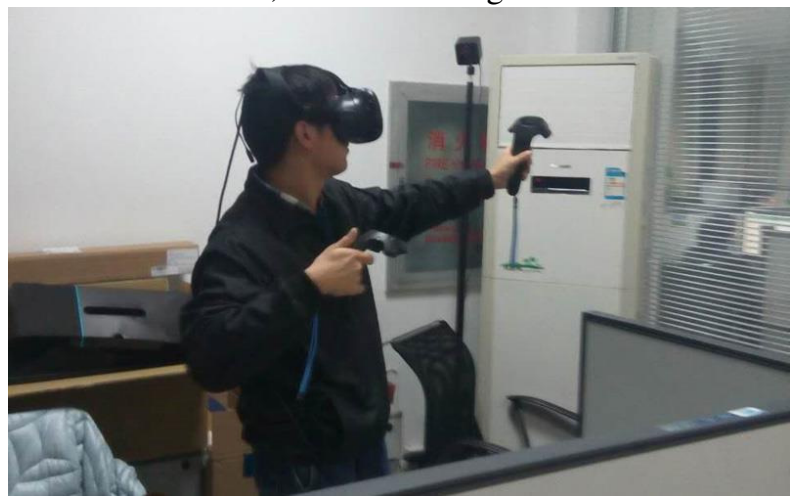

Fig. 3 The view of using HTC VIVE helmet equipment

In the scene shown in Fig. 2, the system track the exact location of the student's hand to determine if it touches the fire extinguisher. The student presses the trigger button on the controller to grab the fire extinguisher. When close to the trash bin, the student presses again to push down the handle of the fire extinguisher and carry out firefighting action. The controllers generate haptic feedback synchronously. Several hand actions are shown in Fig. 4.
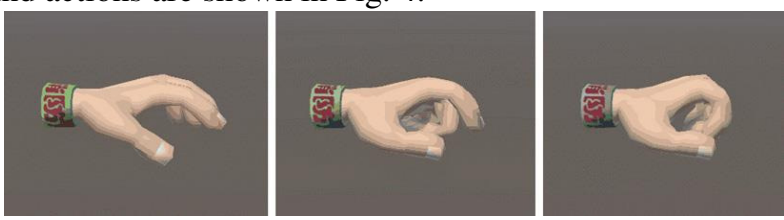

Fig. 4 Hand actions of operating the fire extinguisher

In addition, the necessary guidance and feedback will be provided to make better use of the system and help students correct the error. For example, two yellow footprints and subtitles are set to prompt student to enter the operating area. When the student enters, the color will turn green. When the student is in the wrong direction, the hand tip will appear to guide the student to turn around. 


\section{EXPERIMENT AND ANALYSIS}

\section{A. Usability Test}

The prototype version of fire safety education system has been built and tested. The user feedback is gathered in time to understand the user's comments and suggestions on the system. The usability and effect of the system is analyzed in order to verify that the desired goals have been achieved.

Eight potential users are recruited as testers. They are free to explore in the virtual campus and complete all the learning tasks. Through interviews, the feedback of interface design, ease of operation and process rationality are consulted. The usability test results show that most of them have high degree of satisfaction with the system. They indicate that the system has the characteristics of accessibility, good memorability and high learning efficiency, without fatal errors. Compared to traditional desktop-based interactions (using keyboard and mouse to control virtual devices), the HTC VIVE controllers combined with precise tracking feature can provide better experience, especially in the session of virtual fire drills.

The usability test results are of great help in optimizing the system, and those users feel inconvenient have been further improved. Thus the final solution has been formed.

\section{B. Application Effect Analysis}

Participants in the experiment are randomly recruited on campus, and then 60 students are selected, with the same age, background, and motivation. Through a pre-test volume, they are divided into three groups with the same level, according to the test results. Each group has 10 males and 10 females. The first group is the experimental group, using the VR system based on HTC VIVE. The second group is the control group, using the VR system based on traditional desktop computer. The third group is also the control group, and students use the textbook to learn fire safety knowledge.

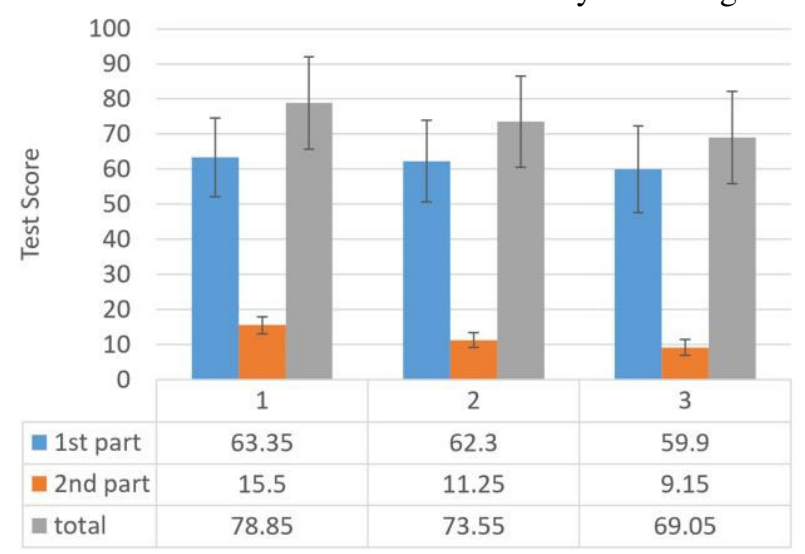

Fig. 5 Mean and variance results of the three groups

Each group uses their own method to learn the fire safety knowledge for 60 minutes, and take 3 rounds of learning process. After that, the three groups are tested within the specified time. The test consists of two parts. The first part is the knowledge quiz, developed by the fire safety knowledge manual issued by government, with full score of 80 points. The second part is operational practice, such as how to use fire extinguishers properly, with full score of 20 points. The judgment is conducted by experienced fire safety teachers. The test results are shown in Fig. 5.

For the first part of the knowledge quiz, the scores of the first and second group are significantly higher than the third group. This shows that the use of VR technology can better improve the effect of fire safety knowledge learning than the textbook teaching. For the second part of the practice, the result of first group is significantly higher than the other two groups. It indicates that students can master more fire safety skills using VR system based on HTC VIVE than traditional system. The experience gained in the virtual environment can be well transferred to actual activities. Overall the VR-based fire safety education system can effectively improve the fire safety learning effect.

\section{V.CONCLUSION}

Fire safety education is very important to every student on campus. The fire safety education system based on virtual reality technology established in the paper has enriched the teaching form of fire safety education on campus, which can effectively help students learn fire safety knowledge, master fire safety skills and improve fire safety education effect.

With the development of modern educational theory and virtual reality technology, it is still necessary to carry out more relevant research in this field.

\section{REFERENCES}

[1] F. M. Williams-Bell, B. M. Murphy, B. Kapralos, A. Hogue, E. J. Weckman, "Using Serious Games and Virtual Simulation for Training in the Fire Service: A Review," Fire Technology, vol. 51. pp. 553-584, May. 2015. DOI: 10.1007/s10694-014-0398-1

[2] W. Viant, J. Purdy, J. Wood, "Serious games for Fire and Rescue training," in 8th Computer Science and Electronic Engineering Conference, pp. 136-139, 2016. DOI: 10.1109/CEEC.2016.7835902

[3] A. J. Houvouras, M. T. Harvey, "Establishing fire safety skills using behavioral skills training," Journal of Applied Behavior Analysis, vol. 47, pp. 420-424, Apr. 2014. DOI:10.1002/jaba.113

[4] J. Bertram, J. Moskaliuk, U. Cress, "Virtual training: making reality work?" Computers in Human Behavior, vol. 43, pp. 284-292, Feb. 2015. DOI: 10.1016/j.chb.2014.10.032

[5] Z. G. Pan, A. D. Cheok, H. W. Yang, J. J. Zhu, J. Y. Shi, "Virtual reality and mixed reality for virtual learning environments," Computers \& Graphics, vol. 30, pp. 20-28, 2016. DOI: 10.1016/j.cag.2005.10.004

[6] C. J. Chen, S. Y. Lau, C. S. Teh, "A feasible group testing framework for producing usable virtual reality learning applications," Virtual Reality, vol. 19, pp. 129-144, 2015. DOI: 10.1007/s10055-015-0263-7

[7] K. Bhagat, W. K. Liou, C. Y. Chang, "A cost-effective interactive 3D virtual reality system applied to military live firing training," Virtual Reality, vol. 20, pp. 127-140, 2016. DOI: 10.1007/s10055-016-0284-x

[8] Z. Xu, X. Z. Lu, H. Guan, C. Chen, A. Z. Ren, "A virtual reality based fire training simulator with smoke hazard assessment capacity," Advances in Engineering Software, vol. 68, pp. 1-8, Feb. 2014. DOI: 10.1016/j.advengsoft.2013.10.004

[9] D. Martinez, J. Y. L. Lawson, J. P. Molina, A. S. Garcia, P. Gonzalez, J. Vanderdonckt, et al., "A framework to develop VR interaction techniques based on OpenInterface and AFreeCA," in IFIP Conference on Human-Computer Interaction - INTERACT, pp. 1-18, 2011. DOI: 10.1007/978-3-642-23765-2 1 